\title{
Stage IIIB Esophageal Adenocarcinoma AJCC v7
}

National Cancer Institute

\section{Source}

National Cancer Institute. Stage IIIB Esophageal Adenocarcinoma A/CC V7. NCI

Thesaurus. Code C87785.

Stage IIIB includes: T3, N2, M0, Any G. T3: Tumor invades adventitia. N2: Metastasis in

3-6 regional lymph nodes. M0: No distant metastasis. (AJCC 7th ed.) 